\title{
Clinical assessment of rhodopsin in the eye Using a standard fundus camera and a photographic technique
}

\author{
U. B. SHEOREY \\ From the Department of Visual Science, Institute of Ophthalmology, London
}

In recent years, the technique of fundus reflectometry (see Weale, 1974), has been shown to be particularly helpful in locating defects in the primary stage of the transduction of light into vision. It has thus been possible to assess the availability of retinal pigment in cases such as night blindness. When the results are considered together with those from complementary electrophysiological or psychophysical tests, a much clearer diagnosis concerning the site of the lesion is possible. The basis of the measurement is the marked change in the absorption spectrum of the visual pigment rhodopsin when it is bleached by strong exposure to light. Thus if a defined part of the retina is bleachedthat is, an imprint or an 'optogram' is formed on the retina-a comparison of the reflectances of the unexposed and exposed areas can be used as an indicator of the availability of pigment in that region. The measurement can be carried out only for a double transit of light through the retinal layer provided that the bleaching of the eye has affected only the retinal pigment.

In most previous clinical studies, complex apparatus was used and the procedure was involved. We have been able to use a simple procedure and a conventional fundus camera, albeit slightly modified. The use of a standard fundus camera and a flash bleaching technique has obvious clinical advantages-for example, the co-operation required of the patient is minimal. These modifications are described in this paper and the use of this technique in assessing the pigment concentration in rod rich parts of the retina is illustrated. These modifications were designed so that they could either be easily removed from the light path in the camera or left in place provided they did not hinder the original application of the camera. As very little machining is required on the main body of the apparatus, its absence from the clinic is short. An approximate assessment of the density contrast of the optogram can be made immediately after developing and fixing the latent image on the photonegative. A value for the density contrast

Address for reprints: U. B. Sheorey, Department of Visual Science, Institute of Ophthalmology, Judd Street, London WCrH 9 QS can be obtained later by means of a microdensitometer. The modified apparatus thus provides a simple procedure for routine and rapid clinical assessment of rhodopsin in the eye.

\section{Method}

The spectral absorption of the visual pigment is different for the bleached and the unbleached states, and the intensity of the light beam which has traversed through the retinal layer containing the pigment will be modified accordingly. We may compare the intensities either from two adjacent areas, one of which is bleached, or from a given area before and after it is bleached. If this difference is due o.ly to a change in the retinal pigment, we can relate the changes in the concentration of that pigment to changes in optical densities. The change in optical density is a function of the wavelength of light used for the measurement; it is largest in bluish-green light for the rod pigment.

The present method uses a photographic technique to record this difference from adjacent areas of the retina after a defined part of the retina has been bleached. In order to relate the recorded density differences to true differences, the photonegative also carries a calibration which is superimposed at the same time as the recording. Thus, distortions which may be introduced during the process of the development of the latent image on the film can be compensated (Arnold, Rolls, and Stewart, 1971).

\section{APPARATUS}

The basic apparatus consists of a standard Zeiss* fundus camera and its associated electronic power supply unit for the xenon flash tube. The paths of the light beams which are used to provide the retinal illumination for general viewing, flash bleaching, and photography are unfortunately largely common. In order to produce an optogram by bleaching a defined part of the retina, an aperture in this light path is required, but it has to be removed for general viewing of the fundus and during photography.

These modifications are illustrated schematically in Fig. I. The original tubular holder which supports the objective lens $\mathrm{L}$ is replaced by a modified *C. Zeiss, Oberkochen, Germany 
housing. The axial length of the housing and the mechanical dimensions at both its ends are exactly the same as for the original tube so that the two can be interchanged. The housing accommodates a moveable carriage which in turn accommodates an aperture-plate. The apertures can be moved along the optical axis by rotation of the shaft $S_{1}$ so that they can be made conjugate with the patient's retina. Either aperture may be brought into the light path by means of the knob $\mathrm{K}_{2}$. A shielded press-switch, $\mathrm{Sw}$, is mounted at the end of $\mathrm{K}_{2}$ to activate the xenon flash $B_{2}$ for retinal bleaching. This switch is wired electrically in parallel with that on the mechanical shutter of the $35 \mathrm{~mm}$ camera. This duplication of the switch is desirable to enable the flash to be activated independently of the camera and to reduce the number of hand movements by the operator during the procedure.

The aperture-plate $P$ carries two apertures, $A_{1}$ for normal viewing of the retina and for photography, and $A_{2}$ for defining the retinal area that is to be bleached (Fig. Ic). The plate is mounted perpendicular to the optical axis of the fundus camera and in the moveable carriage. Both apertures are made from thin sheets of brass and can easily be removed and exchanged for other shapes and sizes-for example, as might be required for the investigation of the density of the retinal pigment along the vertical meridian. The aperture $A_{1}$ is currently $28 \mathrm{~mm}$ diameter, equivalent to a retinal view of $35^{\circ}$. Access to these apertures may be obtained simply by removing the lens $\mathrm{L}$. All internal surfaces of this housing and the additional components which are mounted in the fundus camera are painted matt-black so as to prevent stray reflections within the camera reaching the patient's eye or the recording camera.

$F_{1}$ is the deep-red colour filter that is required in the path of the white light from the bulb $B_{1}$ to provide a safelight for retinal viewing. It is constructed from a sheet of coloured gelatine (Ilford, no. 608) which is clamped between two thin plates of glass and held within a metal plate. A rectangular slot is required in the side panel of the apparatus for the insertion or removal of the filter from the light path. Note that $F_{1}$ does not need to be handled since it does not interfere with either the bleaching or the recording steps. Two miniature lightemitting diodes* are also provided within the housing as additional fixation targets. These diodes are required because the patient cannot see the internal target $T$ once the aperture $A_{1}$ is changed to $A_{2}$ if his centre of fixation is outside the area defined by $A_{2}$. Furthermore, since the paths of the viewing and the bleaching lights are largely common, $T$ is not illuminated when the bluish-green filter is introduced into the light path before

*Motorola, type MLED 20 photography. Few people, however, have any difficulty in maintaining fixation during the brief interval required to introduce the bluish-green filter and photograph the optogram.

The safelight path is combined with the path of the xenon flash light by means of the half-mirror BS. In order to remove the infrared content of the intense xenon-flash, BS was remade from a piece of heat-absorbent glass. The bluish-green colour filter $F_{2}$, which is required during the recording of the optogram, is placed in the light path after BS. The rest position of this filter is out of the light path as it is manually pushed into place immediately before photography and then retracted. Within the same mount, neutraldensity filters may also be incorporated so as to adjust the retinal illumination independently of the power supply to the flash tube. It is thereby possible to achieve a common setting for bleaching and photography, thus considerably simplifying the procedure and avoiding waiting for the electronic power supply to discharge and recharge between changes in settings.

The calibration is superimposed on the film by placing a density-graticule DG in the recess between the film and the mechanical shutter of the camera; therefore, it is only in the path of the light reflected from the fundus. A typical graticule is illustrated in Fig. Id. The part of the film on which the fundus image is recorded is indicated by the broken circle; the inner rectangle indicates the position of the optogram image relative to the graticule and one frame of the film. The graticules are made by exposing a series of strips-for example, (20 $\mathrm{mm}$ long, $2 \mathrm{~mm}$ wide, spaced $2 \mathrm{~mm}$ apart) on to photographic paper*. For present use, the exposure time was simply varied in steps during a trial run and then the appropriate exposure times were selected so as to produce strips of optical densities in the range $0.05-0.15$. The position, size, and orientation of these calibration steps are determined by the particular densitometric investigation that one wishes to perform. For example, for investigations of the changes in the pigment concentration along the vertical meridian, it would be useful to orientate these strips horizontally-that is, perpendicular to the bleaching rectangle. It is essential that an adequate calibration is recorded on the same film in the vicinity of the region of interest.

\section{CALIBRATION OF THE FLASH SOURCE}

The flux incident upon the patient's retina during the flash bleaching and photographic steps was calculated from the radiometric measurements which were taken on the xenon flash. To do so, a model eye was formed in front of lens $\mathrm{L}$ and a *Ilford $\mathrm{N}_{4} \mathrm{E}_{50}$ 

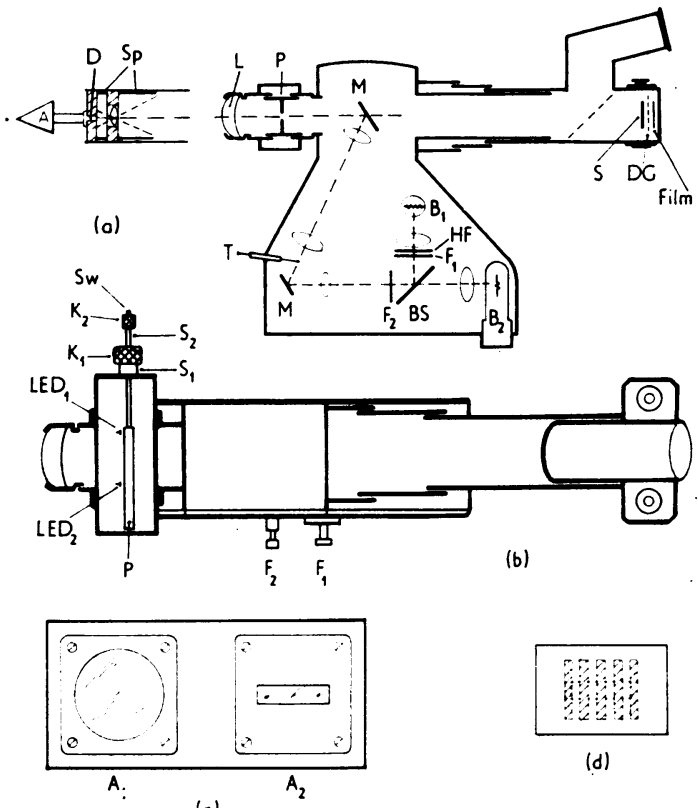

(c)

FIG. I (a) Schematic side view of Zeiss fundus camera showing principal components and modifications.

Tubular housing at front houses objective lens $\mathrm{L}$, and moveable holder for aperture plate $\mathrm{P}$. Also shown are: adjustable, internal fixation target $\mathrm{T}$; mirrors $\mathrm{M}$; combined heat-filter and beam-splitter $\mathrm{BS}$; filter $\mathrm{F}_{2}$ for photographic step; heat-absorbent filter $\mathrm{HF}$; deep-red filter $\mathrm{F}_{1}$; white viewing light $\mathrm{B}_{1}$; xenon flash tube $\mathrm{B}_{2}$; calibration graticule DG; camera shutter $\mathrm{S}$; additional fixation target, LED. Cylindrical assembly to left of lens $\mathrm{L}$ is model eye used to measure flux arriving at simulated retinal plane. During use, assembly is pushed over mounting tube of lens $\mathrm{L}$ and distances are fixed by spacing rings $\mathrm{Sp}$. Output of photodetector $\mathrm{D}$ is fed into amplifier A for measurements

(b) Schematic plan of modified fundus camera showing additional fixation targets LEDs and various control knobs and shafts. Rotation of $\mathrm{K}_{1}$ moves plate holder $\mathrm{P}$ along optical axis for focus adjustments. Bleaching aperture is brought into light path by pulling $\mathrm{K}_{2}$ outwards. Flash can be activated by switch $\mathrm{Sw}$. This is electrically connected to be in parallel with that on shutter release switch of camera

(c) Front view of aperture plate holder $\mathbf{P}$ which carries two apertures, $A_{1}$ for normal viewing of fundus and $\mathrm{A}_{2}$ for bleaching $a$ defined area of fundus

(d) Schematic illustration of density-graticule DG which is placed between the shutter of camera and film so as to impress a calibration on film during photography. Broken line circle, corresponding to aperture $\mathrm{A}_{1}$, indicates area within which retinal image is formed. Inner rectangular region is area, corresponding to $A_{2}$, within which optogram is present photodiode was placed within it. This assembly is shown in Fig. I $a$ to the left of the objective lens L. The lens of the model eye had a focal length of $17 \mathrm{~mm}$ and an aperture of $8 \mathrm{~mm}$ diameter. An objective radiometric method using a photodiode was used in preference to a photometric method using an eye because it is easier and more precise to use routinely in the clinic. The difficulty in making routine photometric measurements by eye with this fundus camera should be noted. The flash source is imaged in the pupil plane of the observer's eye; this image is not uniform and is almost as large as a fully dilated pupil. Furthermore, the output flux of the flash varies according to the interval between firings and the age of the tube.

The waveform of the xenon flash (measured at $\lambda=505 \mathrm{~nm}$ ) consisted of a fast rising front of about $0.1 \mathrm{~ms}$ (measured from 5 per cent of peak to the peak) followed by an exponential decay with a characteristic decay time of about $0.4 \mathrm{~ms}$ (from the peak to I/e of the peak, where $e=2 \cdot 72$ ). For photography of the optogram, the spectral distribution and intensity are defined by the transmittances of the ocular media, the filters, and the optical components within the fundus camera. The retinal energy density during photography is calculated to be $7 \mathrm{Jm}^{-2}$ when using setting no. 4 on the flash control unit and filters $\mathrm{F}_{2}+\mathrm{HF}_{2}+2 \mathrm{E}+0.4 \mathrm{ND}$. The ocular transmittance has been taken as 75 per cent for this calculation; details of this measurement and the calculation are given in the Appendix.

For the bleach, $F_{2}$ and the neutral-density filters are removed from the light path. The increase in the spectral bandwidth and transmittance results in an actinic retinal energy density which is expressed by $T_{e} \times 900 \mathrm{Jm}^{-2}$, where $T_{e}$ is the transmittance of the ocular media. Details of these calculations are also given in the Appendix. Taking a mean value of $T_{e}=60$ per cent over the spectral range $430-630 \mathrm{~nm}$, the bleaching flash is estimated to provide an actinic retinal energy density of $5.4 \times 10^{2} \mathrm{Jm}^{-2}$.

In previous studies on fundus densitometry, the retinal fluxes have been specified in photometric units. These may be related to the values given above by means of the following:

I (scotopic) troland second $=\mathrm{I} \cdot 0 \mathrm{I} \times \mathrm{IO}^{-4} \mathrm{Jm}^{-2}$, for $\lambda=505 \mathrm{~nm}$ and a pupil diameter of $8 \mathrm{~mm}$. Thus, while the value given above for photography compares well with the value of $4 \times 10^{4}$ troland $s$ which was used by Highman and Weale (1973), the bleaching flash currently used is about three times less intense than their figure of $1.82 \times 10^{7}$ troland $s$. The relationship between the fraction of pigment bleached and the retinal illuminance (Fig. 3, Ripps and Weale, 1969) indicates that the present flash will bleach about 55 per cent of the pigment. Thus, if the maximum optical density 
difference that can be achieved in the eyes of normal subjects is taken to be $0.16-0.18 \mathrm{D}$, we may expect to measure a density difference of $0.09-0.10 \mathrm{D}$ in these subjects with the present equipment.

The alignment and focus adjustment of the camera were carried out by using the red filter $F_{1}$ in the path of the white light from the bulb $B_{1}$. On setting 2 of the Zeiss power supply the retinal illumination was calculated to be 350 troland. The luminance of the image of the source formed in the pupil of the model eye was measured by means of a photometer*. The luminances of the additional LED fixation targets were adjusted by varying their input power so that they appeared to have the same luminosity as the surrounding field. The power spectrum of these LEDs is negligible at wavelengths shorter than $630 \mathrm{~nm}$ and their emission is confined to a narrow band in the deep red part of the spectrum.

\section{Procedure}

The patient is seated at the fundus camera in a darkened examination room. The pupil must have been fully dilated and dark adaptation allowed to proceed for at least $15 \mathrm{~min}$ before this. The plane of the film and the apertures are adjusted rapidly so as to be in focus with the retina. Since it is difficult for the operator to see much retinal detail in the dim red light, it is helpful to begin the adjustments while viewing the region around the optic disc which has a larger reflectance and is rich in structure. Note that a slight re-adjustment will be necessary to view different parts of the fundus but no correction for chromatic aberration is required. The required retinal area is then brought into the centre of the field of view with the moveable internal fixation target or one of the LED targets and the focus checked. A retinal area in which there are relatively few blood vessels should be selected as far as possible, so that the traces recorded by the scanning densitometer are smooth and thus easier to analyse. The aperture $\mathbf{A}_{2}$ is brought into the optical path by pulling the shaft $S_{2}$, the xenon flash is activated by means of the switch Sw, and the shaft is pushed back; this completes the bleaching step. If the eye is still correctly placed, the filter $F_{2}$ is pushed into the light path, the retina is photographed, and $F_{2}$ is pulled back to its resting position. The camera shutter is set nominally to $\mathrm{r} / 25 \mathrm{~s}$. The film is then advanced by one frame so that the camera is ready for another photograph or the next sequence. $A$ readily available sensitive film $\dagger$ is used for this reading. The film is processed in complete darkness and according to the manufacturer's instructions.

Careful alignment of the patient's eye to the apparatus is particularly important in this technique, because it is essential to transmit all of the available flux into the patient's eye for bleaching. During photography, the spurious reflections and scatter from the eye that can so easily mask the image of the optogram, must be *Model 40A, made by UDT Inc, California, USA

†Kodak 2475 avoided. Note that with the subject's fixation on the periphery of the apertures $A_{1}$ or $A_{2}$, the pupil will appear to be elliptical as viewed along the optical axis of the apparatus. Thus there is a reduced margin for the transverse alignment of the image of the light source, formed in the subject's pupil. A misalignment would mean that a part of the beam would be occluded and this could cause a serious reduction in the retinal of illumination. For a given patient, the focus and the fixation target require little, if any, adjustments on recalling the patient after a period of rest or further dark-adaptation. In order to ensure accurate fixation during the course of this procedure, the fixation target should be presented to the same eye as that used for recording the optogram.

\section{Results and the analysis of the optogram}

A positive print of a typical retinal photograph taken with this modified fundus camera is shown in Fig. 2. This was recorded from a subject in whom dark-adaptation and vision were normal. The rectangular optogram can be clearly seen in the centre of this photograph as an area of lower optical density. This area is crossed by the regularly spaced stripes caused by superimposition of the density graticule. It is important to note that the optical densities are reversed during the process of this photographic printing-that is, the bleached area in reality reflects more bluish-green light than the surrounding unbleached region so that it is more dense on the photonegative. The aperture $A_{2}$ used for bleaching was centred about $15^{\circ}$ temporal to the fovea, along the horizontal meridian. The backs of the two LED fixation targets which

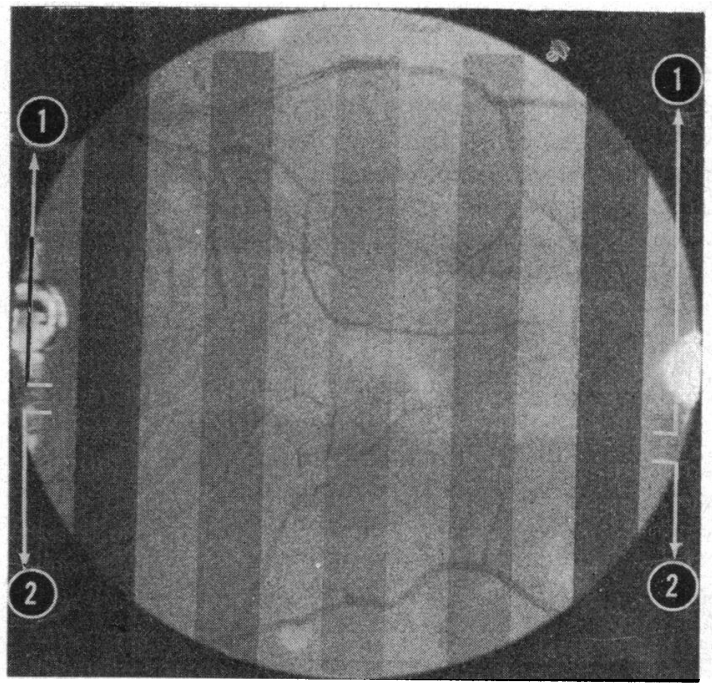

FIG. $2 A$ positive photographic print of typical optogram recorded from normal fundus. Subject's point of fixation is marked by LED target at left edge 
are provided within the lens housing can also be seen; the subject's fixation is marked by the illuminated LED target at the left of the photograph.

In order to obtain quantitative results from the fundus photograph, the photonegative has to be scanned in a microdensitometer; a double-beam recording microdensitometer* was used for the present work. The displacement of the tracerecorder, due to a change in the optical transmittance of the scanned sample, has to be initially calibrated; this was done by introducing known values of neutral-density filters in the path of the measurement beam. Then, using common experimental conditions for both measurements-for example, the same spot size and scan rate-the density-graticule and the photonegative are scanned in turn. The difference between the scans taken through the bleached area and the adjacent unbleached area is proportional to the true optical density difference. The proportionality constant is the transfer characteristic of the particular part of the film (Arnold and others, I97I). For a given region of the film if the step change due to the superimposed density graticule is the same for both scans, then this constant is unity. Thus deviations from unity can be noted and the results scaled accordingly. The procedure can best be illustrated by referring to Fig. 3 which shows the traces (corresponding to the scans taken through the sections indicated in Fig. 2). The traces are drawn about a datum line which is taken to be the base or fog level of the film. A scan of the particular density graticule which was used for this photograph is also shown in Fig. 3. Note, however, that this third trace has been drawn inverted so as to illustrate the point that the exposure received by the film which lies under the calibration strips is reduced. This local reduction of exposure can also be seen in the other two traces which are pulled down towards the datum in the regions containing the calibration strips. The calibration for the graticule is indicated in the centre of this figure. The transfer characteristic of this photonegative can'be

*Model 3c, made by Joyce-Loebl Ltd seen to be slightly above unity. The density contrast of the optogram formed in central part of this photograph-that is, $15^{-20^{\circ}}$ temporal-is about $0 \cdot 1$ D. The density difference can be determined more easily, in practice, when all three traces are available on separate sheets of transparent graph paper. The sheets containing the scans taken outside and through the bleached region are then viewed against the sheet for the graticule. The graticule is once again held inverted and used as a template.

These traces illust:ate several additional interesting features. The traces are noisy and the reflectance varies across the retira. This variation is due to a variety of reasons, partly in the instrument and partly in the subject's eye-for example, the presence of blood vessels in the path of the scan or a non-uniform retinal reflex. Note, however, that the scale of this variation is either much smaller or else much larger than that due to the modulation impressed by the graticule on the fundus photograph. Thus it is easy to discriminate between the true density changes and the artefacts. The traces also illustrate the variaticn of the density difference across the retina. This variation is practically zero for the part of the retina nearer than $10^{\circ}$ temporal to the fovea. It rises rapidly beyond this to a broad maximum in the region $15-30^{\circ}$ temporal, in general agreement with the distribution of rods in the retina. For routine clinical investigations, one would be interested chiefly in the central portion of the photograph. Thus, as indicated above, a graticule is made so that it provides a density step of a value which is of the order of the density difference that one expects to measure from normal subjects. A marked reduction in the density difference recorded in a patient's eye would thus be readily seen to be much smaller than this step. The two outermost strips are well outside the expected range. However, they illustrate the fact that even when the illumination on the film is reduced by 0.35 density units, an appreciable density difference can still be recorded. This difference can of course be achieved only from optograms formed in regions

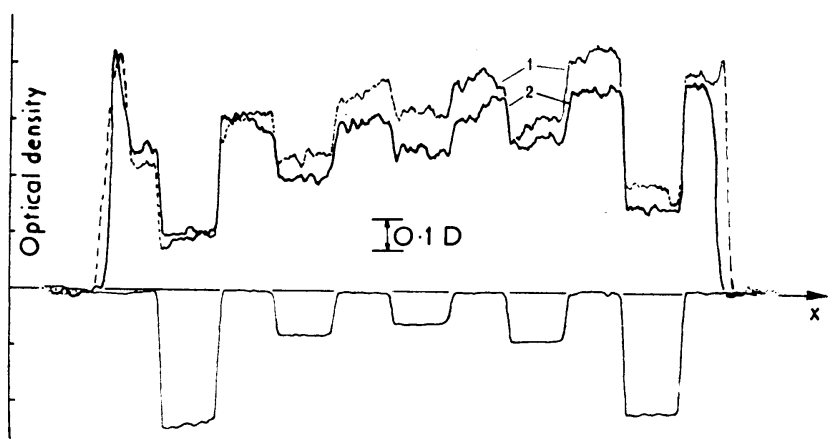

FIG. 3 Trace recordings produced by scanning microdensitometer. Traces $\mathrm{I}$ and 2 are due to scans taken through corresponding sections which are indicated in Fig. 2. Lowest trace is scan of particular density graticule used to superimpose calibration. Scan has been drawn inverted so as to indicate that exposure of film underlying density strips is reduced. Ordinate represents optical density; abscissa represents distance along horizontal meridian on fundus 
of the retina distal from the macula. Thus the film as such appears to have an operational range which encompasses the gross variations of retinal reflex across the retina. Note also that in this graticule the densities of the steps increase away from the vertical midline. Thus a graticule such as this may be used to photograph the optogram in corresponding regions of either eye without requiring the replacement of graticules during measurements on both eyes.

\section{Discussion}

This example of the use of the modified fundus camera was typical; a clinical study of various retinal disorders using this apparatus is in progress and full results will be described in due course. Thus the comments below refer mainly to points of technique. In regular use, this modified fundus camera has proved to be no more difficult to use than is the normal camera for routine fundus photography. As the method provides a rapid, convenient means of supporting the measurements of associated retinal tests in diseases such as nightblindness, routine densitometric measurements may also be carried out in the same clinic with this apparatus.

The maximum flux available from the present equipment is insufficient to bleach all the visual pigment within a given region. The flux could be increased but only by modification, perhaps extensive, of the optics or the flash unit. However, as established by Highman and Weale (1973) in cases such as retinitis pigmentosa, the density difference 3 in a given retinal region are much lower, and the vis aal thresholds of the same retinal region are much higher, than those in normal subjects. Therefore, for the assessment of such cases the apparatus may be used without any further modifications. It should be noted that simply increasing the incident flux may not give a markedly denser optogram, because short intense flashes of light can bleach only up to a limit of 70-80 per cent of the available pigment because of photoregeneration (Williams, 1974). Furthermore, the associated heating effect of the intense retinal illumination may well produce a deleterious effect-that is, oedema. The optical effect of this oedema is partially to counteract the density difference due to the bleach (Weale, 1968). Its magnitude, however, is said to be independent of the wavelength of light used for the measurement so that it may be quantified by measurements outside the absorption spectrum of the pigment. In order to ascertain whether oedema had been caused, a series of four or five fundus photographs were taken in deepred light immediately after the retina had been bleached and at half-min intervals subsequently.
The filter $F_{2}$ was replaced by a suitable deep-red colour filter*. In these photographs, no density difference corresponding to the bleaching aperture could be seen in the region of the optogram. The experimental conditions in the earlier fundus densitometric measurements were markedly different from those used currently-for example, Highman and Weale (1973) used a continuous io s intense exposure for bleaching - and therefore comparative assessments of the various procedures are difficult to make. In the previous studies, only the photometric effect of the bleaching light was specified, whereas in the present approach, both the actinic and the total retinal flux densities are estimated. Thus the total flux entering the eye can also be calculated (see Appendix).

This apparatus cannot be used in its present form at large viewing angles-that is, no larger than about $15^{\circ}$ in either the vertical or the horizontal meridians. In order to do so, the image of the flash source which is formed in the subject's pupil would need to be reduced without reducing the incident flux. This reduction in the pupillary area as a function of the viewing angle was mea sured by Jay (1961). A smaller pupillary image would additionally reduce the variation in the retinal illumination due to the involuntary eye movements. These are particularly noticeable in older patients in whom more care is required for alignment. Note that a slight variation in the retinal illuminationfor example, as a result of ocular absorption or small eye movements-does not restrict the use of this reflectometric method of measurement because a differential measurement is involved. The reduction in the retinal illumination during the bleaching step, however, could be significant since the density contrast of the optogram would be reduced. The reduction in the flux as a result of absorptionfor example, in the lens-could be compensated by an appropriate increase in the incident flux using the data established by Said and Weale (1959), or by a specific measurement in a particular case.

\section{Summary}

A technique based on the method of differential fundus reflectometry is used to assess the availability of rhodopsin in the eye. A defined part of the dark adapted fundus is bleached by a short intense flash of light. The fundus is subsequently photographed in order to record the flux reflected from the bleached area, the optogram, and the surrounding unbleached region. This procedure requires only a few simple modifications to a Zeiss fundus camera before it can be used routinely in the clinic.

*Ilford No. 608 
The use of the Zeiss fundus camera for fundus densitometry was suggested by Professor R. A. Weale whose keen interest during the modifications and the clinical trials was invaluable. The modifications to the camera were carried out by Mr G. Mould.

Thanks are also due to $\mathrm{Mr} \mathrm{P}$. E. Cleary for collaboration with the clinical development.

\section{Appendix}

\section{MEASUREMENT AND ASSESSMENT OF THE RETINAL FLUX}

The absorbances $A_{\lambda}$ of the various spectral filters used in the procedure and for calibration were measured in a dual-beam scanning spectrophotometer*. The results are tabulated below:

\begin{tabular}{ll}
\hline Filter & $\begin{array}{l}\text { Absorb- } \\
\text { ance } \\
\text { (density) }\end{array}$ \\
\hline $\mathrm{F}_{2}$ & $\begin{array}{l}0.60 \\
\mathrm{HF}_{2}\end{array}$ \\
Wratten $_{2} \mathrm{E}$ & $\begin{array}{l}0.07 \\
0.03\end{array}$
\end{tabular}

The flux density in the retinal plane which was simulated within the model eye was measured by a fast-response photodiode $\dagger$. The photodiode was operated in the photoconductive mode, using a bias of $-45 \mathrm{~V}$ on its guardring electrode. The photocurrent was fed directly into an operational amplifier which was constructed from an integrated circuit, type LM 308. This was wired so as to perform either as a linear current-to-voltage converter or an integrator. Thus the envelope of a photocurrent pulse resulting from the detection of a xenon flash could be viewed on an oscilloscope or the integral of the current pulse could be measured by means of a voltmeter. The linearity of the operational-amplifier circuit was checked by injecting pulses of current from a pulse generator and through suitable known values of resistors. The response time (measured between the ro per cent and 90 per cent *Perkin Elmer, model 402

†SGD rooA, a silicon PIN type, supplied calibrated by E. G. and G. Inc, Boston, USA amplitude points) was less than $20 \mu \mathrm{s}$, a value considerably less than the rise-time of the xenon flash. A capacitor of ro $\mu$ Farad was used in the feedback path of the operational amplifier in order to measure the total charge of one pulse of photocurrent. Using $\mathrm{F}_{2}+\mathrm{HF}_{2}+2 \mathrm{E}+\mathrm{I} \cdot 3 \mathrm{ND}$ filters within the light path of the fundus camera and setting no. 4 on the flash control unit, the output was $130 \pm 3.7 \mathrm{mV}$ (mean of five flashes, ignoring the first flash). Thus the charge accumulated was $1 \cdot 3 \times 10^{-6} \mathrm{C}$ per flash. The response sensitivity of the photodiode is $0.217 \mathrm{~A} / \mathrm{Watt}$, at $\lambda=505 \mathrm{~nm}$, and the active area is $5.1 \times 10^{-6} \mathrm{~m}^{2}$. Thus the energy density received by the photodiode at the simulated retinal plane is $1 \cdot 18 \mathrm{Jm}^{-2}$. Note that when the flash is first fired after a rest period of a few minutes, the flux from the first flash is about 5 per cent higher than that of the subsequent flashes which are fired at regular intervals of, say, 5 or ro s.

During the retinal photography step, the filters $\mathrm{F}_{2}+\mathrm{HF}_{2}+2 \mathrm{E}+0.4 \mathrm{ND}$ are used in the fundus camera. Taking the transmittance of the ocular media as 75 per cent at $\lambda=505 \mathrm{~nm}$ (Table 2.7, Wyszecki and Stiles, 1967) the retinal energy density is $7.04 \mathrm{Jm}^{-2}$. The retinal energy density may then be calculated for any given combination of filters inserted in the fundus camera, given the spectral distribution of the xenon flash-discharge source. Although there are several narrow peaks in the $460-490 \mathrm{~nm}$ band, in most applications the distribution may be taken to be essentially flat over the range $400-630 \mathrm{~nm}$. Thus the use of a broad spectral band for bleaching, say, from 430-630 nm, increases the total flux by about 300 times more than that used during photography. If we take the mean transmittance of the ocular media as 70 per cent, the retinal energy density within this spectral band is about $\mathrm{I} \cdot 5 \times 1 \mathrm{O}^{3} \mathrm{Jm}^{-2}$.

In order to calculate the increase in the actinic flux during the bleaching step, the absorption spectrum of rhodopsin was used (Crescitelli and Dartnall, 1953). In the spectral range $430-630 \mathrm{~nm}$, the increase is 9.6 times more than that in a $10 \mathrm{~nm}$ bandwidth centred at $505 \mathrm{~nm}$. Thus, the removal of the neutral density filter and $\mathrm{F}_{2}$ from the light path.will increase the actinic flux at the retinal plane to: $1 \cdot 18 \times 9.6 \times \log _{10}{ }^{-1}(\mathrm{r} \cdot 9) \times \mathrm{T}_{\mathrm{e}}$ or $900 \times \mathrm{T}_{\mathrm{e}} \mathrm{Jm}^{-2}$.

\section{References}

ARNOld, C. R., Rolls, P. J., and Stewart, J. C. R. (197r) 'Applied Photography', Focal Press, London CRESCITELli, F., and DARTNALl, H. J. A. (1953) Nature (Lond.), 172, 195 highman, v. N., and Weale, R. A. (1973) Amer. F. Ophthal., 75, 822

JAY, B. (196r) Vision Res., $\mathrm{r}, 4 \mathrm{r} 8$

RIPPS, H., and Weale, R. A. (1969) $\mathcal{F}$. Physiol. (Lond.), 200, I 5 I

SAID, F. S., and WEALE, R. A. (I959) Gerontologica (Basel), 3, 213

WEALE, R. A. (1968) Nature (Lond.), 218, 238

(1974) Ophthalmologica (Basel), 169, 30

Williams, T. P. (1974) Vision Res., 14, 603

WYSzEcki, G., and STILES, W. s. (r967) 'Colour Science', Wiley, New York 